\title{
Stem Cell
}

National Human Genome Research Institute (NHGRI)

\section{Source}

National Human Genome Research Institute (NHGRI). Stem Cell.

A stem cell is a cell with the potential to form many of the different cell types found in the body. When stem cells divide, they can form more stem cells or other cells that perform specialized functions. Embryonic stem cells have the potential to form a complete individual, whereas adult stem cells can only form certain types of specialized cells. Stem cells continue to divide as long as the individual remains alive. 